\title{
Decrease in lipid rich plaque during percutaneous coronary intervention: reality or artefact? An observational study using Near-Infrared Spectroscopy
}

\begin{abstract}
Background: Lipid rich plaque (LRP) content has been shown to decrease after high-pressure balloon-expandable stent placement. LRPs are quantified by the lipid core burden index (LCBI) on near-infrared spectroscopy (NIRS). We aim to evaluate the changes in LRP content during sequential steps of non-urgent percutaneous coronary intervention $(\mathrm{PCl})$ using low-pressures.
\end{abstract}

Methods: A total of 10 patients with a LCBI>200 on pre-stenting NIRS and successful PCI with the self-apposing stent were included. Quantitative coronary angiography was used to assess luminal diameters. IVUS-NIRS was performed pre-stenting after initial pre-dilatation, post-stenting and after post-dilatation to assess the LCBI and the plaque burden to evaluate plaque modification. IVUS-NIRS is analyzed for all stented segments including proximal and distal stent edges using dedicated software.

Results: Mean balloon pressure was $11.4 \pm 3.8$ atm. Pre-stent reference vessel diameter (RVD) was $3.3 \pm 0.6 \mathrm{~mm}$, Dmax was $3.9 \pm 0.9 \mathrm{~mm}$. Mean LCBI of the stented segment was $160 \pm 96$ pre-stenting, $32 \pm 49$ post-stenting and $21 \pm 35$ after post-dilatation $(p=0.001)$. The plaque burden remained constant in the stented segment; $0.48 \pm 0.1 \mathrm{~mm}^{2}$ pre-stenting vs. $0.51 \pm 0.1 \mathrm{~mm}^{2}$ post-stenting vs. $0.48 \pm 0 \mathrm{~mm}^{2}$ after final post-dilatation $(\mathrm{p}=0.14)$.

Conclusions: Serial IVUS-NIRS demonstrated a significant decrease of LCBI in the stented segment from pre-stenting to post-stenting and after post-dilatations, while plaque burden remained unaltered throughout $\mathrm{PCl}$ in relatively large RVDs. This discrepancy might possible explained by the fact that NIRS might be not detecting the lipid in larger RVDs since it is only validated in small RVDs. Research including larger numbers of patients are warranted to confirm this concept.

Keywords: Plaque modification - Near-Infrared Spectroscopy - Coronary artery disease defects Stent endothelization

\section{Introduction}

Atherosclerotic plaques containing a large lipid core, called lipid rich plaques (LRP), can be prone to imminent plaque rupture, and predictive for future cardiovascular events [1-5]. Many of those LRP are treated with stenting. There is an increased risk of procedural related complications during percutaneous coronary intervention (PCI) involving lesions containing large LRPs.
These complications may include plaque shift and distal embolization of plaque debris, which could impair clinical outcomes [6]. There is emerging evidence that the reduction of the lipid content of LRPs is associated with the occurrence of distal embolization [7-10].

The combined intravascular ultrasound (IVUS) and near-infrared spectroscopy (NIRS) catheter system has been validated to identify the chemical composition of the
Huangling $L u^{1}$, Ruben YG Tijssen', Maik J Grundeken', Robin P Kraak', Jan GP Tijssen"1, Robbert J de Winter ${ }^{1}$, Karel T Koch', Hector M Garcia-Garcia ${ }^{2,3}$ and Joanna J Wykrzykowska1* 'Department of Cardiology, Academic Medical Center, University of Amsterdam, Amsterdam, the Netherlands

${ }^{2}$ Cardiovascular Core Lab, MedStar Health Research Institute, Washington DC, United States of America 3Angiographic Core Lab, Cardialysis BV Rotterdam, the Netherlands

*Author for correspondence:

Tel: +31205665204

E-mail: j.j.wykrzykowska@amc.nl

Submitted: November 14, 2017

Accepted: December 07, 2017

Published online: December 11, 2017 
arterial wall and to perform a morphometric assessment, including modification of LRPs throughout PCI [11]. With the introduction of the STENTYS self-apposing nitinol coronary stent, which has the property to oppose itself to the arterial wall and could theoretically be deployed with reduced procedural pressures, the risk of plaque shift during the procedure and distal embolization of plaque debris might be reduced. The nitinol stent platform of the STENTYS stent adjusts to the vessel anatomy without exerting a large outward radial force, which is seen with the implantation of balloon expandable stents.

We therefore performed an observational study to evaluate the changes in lipid content and plaque burden during PCI with the use of the self-apposing stent, using IVUS-NIRS pre-stenting, directly after stent implantation, and after final post-dilatations.

\section{Materials and Methods}

\section{Patient population}

This study was a prospective single center observational study performed in the Academic Medical Center (AMC), University of Amsterdam, the Netherlands, a high-volume, tertiary PCI center. This study evaluated the differences of lipid content and plaque burden at sequential stages during PCI with a self-expanding STENTYS stent. Elective patients were eligible if they had a de novo coronary artery stenosis and a lesion that could be treated with a single stent. The indication for the STENTYS stent, as set by the local heart team, was based on the suitability of the lesion for the STENTYS stent. The study is approved by the local ethics committee. Written informed consent was obtained, before any study-related procedure took place.

\section{Study device}

The STENTYS stent is a self-expanding stent made of a nitinol platform, a biocompatible nickel and titanium alloy. It is deployed by withdrawing a retractable sheath, thus no balloon is used to expand the stent with this conventional delivery system. Therefore, the stent is placed with a gentle outward force, although in most cases it is still necessary to post-dilate the stent after placement to ensure optimal expansion. The STENTYS stent received Conformité Européene mark in 2010 and is used in our center both in daily clinical practice as in clinical trials. The available device diameter sizes were: small suitable for vessel diameters between 2.5 and 3.0 $\mathrm{mm}$, medium for vessel diameters between 3.0 and 3.5 $\mathrm{mm}$, and large for vessel diameters ranging from 3.5 to
$4.5 \mathrm{~mm}$. The large STENTYS is capable to expand up to $6.4 \mathrm{~mm}$, which provides adequate apposition even in the cases of very large vessels. The available device lengths were $17 \mathrm{~mm}, 22 \mathrm{~mm}$ and $27 \mathrm{~mm}$. The stent is available as BMS and DES (Sirolimus-eluting). The novel balloon based delivery system was not yet available for commercial use during our study.

\section{Study procedure}

The procedure involving the STENTYS stent was performed in the setting of routine clinical care. Study procedures, including serial intracoronary IVUS-NIRS image acquisition, were performed during the PCI only within the target vessel. The combined grayscale IVUS and NIRS imaging system consists of a 3.2 French rapid exchange catheter (LipiScan, InfraReDx, Burlington, MA, USA) with pullback and rotation device for IVUS and NIRS co-registration and a motorized console. Serial motorised pullbacks were performed at a pullback speed of $0.5 \mathrm{~mm} / \mathrm{second}$. The imaging catheter was advanced at least $10 \mathrm{~mm}$ distally from the target lesion if possible.

Serial IVUS-NIRS pullbacks of the culprit lesion were performed at 3 moments during PCI:

1. Pre-stenting, after pre-dilatations before stent placement (pre-stent);

2. Post-stenting, directly after stent implantation (post-stent);

3. Final, after final post-dilatation (final); i.e. until residual stenosis is less than 20 percent, according to the instructions for use. If additional post-dilatations were needed, an additional IVUS-NIRS pullback was obtained after the final post-dilatation.

Angiographic success was defined as a final residual stenosis of less than 20 percent by visual estimation and TIMI 3 flow on the final angiogram. Procedural success was defined as angiographic success without in-hospital major adverse cardiac events (composite of cardiac death, any myocardial infarction or target vessel revascularization).

\section{Quantitative coronary angiography}

Quantitative coronary angiography (QCA) analyses were performed pre-stenting, post-stenting and after final balloon dilatation. Analyses were performed with dedicated software (QAngioXA version 7.3; Medis, Leiden, The Netherlands). A standardized QCA methodology was used. Matched angiographic views pre- and post-procedural were used to calculate the 
reference vessel diameter (RVD), minimal luminal diameter (MLD) and the maximal luminal diameter (Dmax).

\section{Intravascular ultrasound and Near-Infrared Spectroscopy analysis}

IVUS-NIRS data were analysed using dedicated offline software (QIVUS version 3.0, Medis, Leiden, The Netherlands). Derived chemograms were analysed to assess the maximum lipid core burden index (LCBI) in the following segments; 1 ; the proximal $5 \mathrm{~mm}$ of the stent edge, 2; the stented segment and 3; the distal 5 $\mathrm{mm}$ of the stent edge (Figure 1). Lumen and external elastic membrane (EEM) contours were drawn at each IVUS cross section from $5 \mathrm{~mm}$ proximal to the stent edge, at the proximal and distal stent edges, to $5 \mathrm{~mm}$ distal to the stent edge, and at the first and last frame of each segment. An interval of $2 \mathrm{~mm}$ was taken in between contours. Target segments were matched using anatomical landmarks including side branches, veins and calcification spots. Plaque and media cross-sectional area (CSA) were defined as the difference of EEM CSA minus lumen CSA (in $\mathrm{mm}^{2}$ ). Plaque burden was defined as plaque and media CSA divided by the EEM CSA. Each analysed sub-segment had IVUS-derived plaque burden and NIRS-derived LCBI measured to evaluate the plaque modification and lesion remodeling during and after PCI. All IVUS-NIRS pullbacks were analysed by two independent assessors (H.L., R.T) and were validated by an expert assessor (H.G.).

\section{Statistical analysis}

Continuous variables were presented as mean \pm standard deviation (SD), categorical variables as frequencies (percentage). To compare groups, the paired Student T test or the one-way ANOVA test were used where appropriate. Correction for sphericity was performed with the Greenhouse-Geisser if necessary.
All data were statistically analyzed using SPSS software package (version 23, IBM, Chicago, IL, USA).

\section{Results}

\section{Patient and procedural characteristics}

A total of 12 consecutive patients with a STENTYS indication who were eligible by the local heart team between May 2013 and July 2014 were included in this study. Two patients were excluded from the analyses, since they had a $\mathrm{LCBI}<200$ on the pre-stenting IVUS-NIRS pullback. Baseline patient characteristics are summarized in Table 1 . Out of 10, nine patients underwent PCI in a native coronary artery and 1 patient in a saphenous vein graft (SVG). Pre- and postdilatation was performed in all patients, with maximal pressures of $11.4 \pm 3.8 \mathrm{~atm}$ (pre dilatation) and 11.1 \pm 2.5

\begin{tabular}{|lc|}
\hline Table 1: Baseline characteristics. & \\
\hline Patient characteristics & $\mathrm{n}=10$ \\
\hline Age & $64 \pm 10$ \\
\hline Male gender, $\mathrm{n}(\%)$ & 8 \\
\hline Diabetes, $\mathrm{n}(\%)$ & 0 \\
\hline Hypertension, $\mathrm{n}(\%)$ & 5 \\
\hline Hypercholestrerolemia, $\mathrm{n}(\%)$ & 2 \\
\hline Current smoker, $\mathrm{n}(\%)$ & 3 \\
\hline Positive family history, $\mathrm{n}(\%)$ & 9 \\
\hline Previous MI, $\mathrm{n}(\%)$ & 1 \\
\hline Previous PCl, $\mathrm{n}(\%)$ & 2 \\
\hline Previous CABG, $\mathrm{n}(\%)$ & 1 \\
\hline Previous Stroke, $\mathrm{n}(\%)$ & 2 \\
\hline Congestive heart failure, $\mathrm{n}(\%)$ & 0 \\
\hline Peripheral artery disease & \\
\hline Renal insuffiiency & \\
\hline Indication for PCl & 5 \\
\hline Stable angina & 1 \\
\hline Unstable angina & 4 \\
\hline Non STEMI & \\
\hline
\end{tabular}
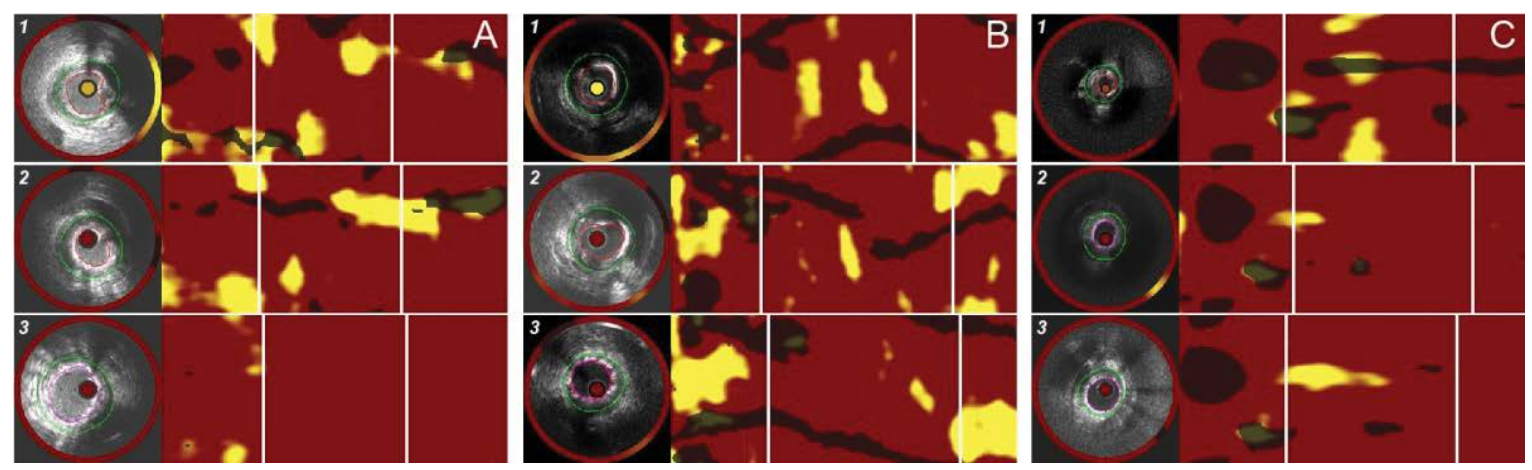

Figure 1: Longitudinal NIRS imaging analyses. IVUS NIRS chemogram (1) pre-procedural chemogram, (2) post-stenting chemogram, (3) final chemogram; in panel A and B patients with stable angina and in panel C a patient with NSTEMI demonstatring the lipid shift within the stented vessel segment. 
atm (post dilatation). Pre-dilatation balloon diameters were $2.9 \pm 0.2 \mathrm{~mm}$, post-dilation balloon diameters were $3.9 \pm 0.4 \mathrm{~mm}$. In 1 patient $(10 \%)$ a Spider distal embolic protection device was used during the procedure. Pathologic analysis from the obtained material with this device revealed that the material was $3 \mathrm{~mm} 2$ of dried thrombus, without any evidence of lipid. Angiographic and procedural success was both $100 \%$. Procedural characteristics are summarized in Table 2.

\section{Angiographic outcomes}

Mean pre-stent RVD was $3.3 \pm 0.6 \mathrm{~mm}$. Mean MLD was $0.9 \pm 0.4 \mathrm{~mm}, 2.2 \pm 0.2 \mathrm{~mm}$ post-stenting and $3.0 \pm 0.4 \mathrm{~mm}$ after post-dilatation $(\mathrm{p}<0.001)$. The preprocedural maximal diameter (Dmax) was on average $3.9 \pm 0.9 \mathrm{~mm}$ with a maximum of $5.00 \mathrm{~mm}$ (Table 3 ).

\section{IVUS-NIRS outcomes}

The results of the IVUS- NIRS analyses are summarized in Table 3. In the stented segment, the MLA was $5.2 \pm 1.2 \mathrm{~mm}^{2}$ pre-stent, $4.7 \pm 1.2 \mathrm{~mm}^{2}$ poststent and $8.4 \pm 2.4 \mathrm{~mm}^{2}$ final $(\mathrm{p}<0.001)$. LCBI within

\begin{tabular}{|c|c|c|}
\hline \multicolumn{2}{|l|}{ Location of study lesion } & $L=10$ \\
\hline \multicolumn{2}{|l|}{ LAD } & 3 \\
\hline \multicolumn{2}{|l|}{$\mathrm{RCx}$} & 1 \\
\hline \multicolumn{2}{|l|}{ RCA } & 5 \\
\hline \multicolumn{2}{|l|}{ SVG } & 1 \\
\hline \multicolumn{3}{|c|}{ ACC/AHA lesion classification } \\
\hline \multicolumn{2}{|l|}{ A } & 1 \\
\hline \multicolumn{2}{|l|}{ B1 } & 1 \\
\hline \multicolumn{2}{|l|}{ B2 } & 8 \\
\hline \multicolumn{2}{|l|}{ C } & 0 \\
\hline \multirow[t]{3}{*}{ Stent length $(\mathrm{mm})$} & $17 \mathrm{~mm}$ & 1 \\
\hline & $22 \mathrm{~mm}$ & 7 \\
\hline & $27 \mathrm{~mm}$ & 2 \\
\hline \multirow[t]{3}{*}{ Stent diameter size $(\mathrm{mm})$} & $2.5-3.0 \mathrm{~mm}$ & 0 \\
\hline & $3.0-3.5 \mathrm{~mm}$ & 4 \\
\hline & $3.5-4.5 \mathrm{~mm}$ & 6 \\
\hline \multicolumn{2}{|l|}{ Pre-dilatation performed } & 10 \\
\hline \multicolumn{2}{|c|}{ Pre-dilatation max pressure (atm) } & $11.4 \pm 3.8$ \\
\hline \multicolumn{2}{|l|}{ Pre-dilatation diameter } & $2.9 \pm 0.2$ \\
\hline \multicolumn{2}{|l|}{ Post-dilatation performed } & 10 \\
\hline \multicolumn{2}{|c|}{ Post-dilatation max pressure (atm) } & $11.1 \pm 2.5$ \\
\hline \multicolumn{2}{|l|}{ Post-dilatation diameter } & $3.9 \pm 0.4$ \\
\hline \multicolumn{3}{|c|}{ Use of distal embolization protection device 1} \\
\hline \multirow[t]{4}{*}{ Postprocedure TIMI flow } & Grade 0 & 0 \\
\hline & Grade 1 & 0 \\
\hline & Grade 2 & 0 \\
\hline & Grade 3 & 10 \\
\hline \multicolumn{2}{|l|}{ Angiographic success } & 10 \\
\hline \multicolumn{2}{|l|}{ Procedural success } & 10 \\
\hline
\end{tabular}

the stented segment was $160 \pm 96$ pre-stent, $32 \pm 49$ poststent and $21 \pm 35$ final $(\mathrm{p}=0.001)$. The LCBI within
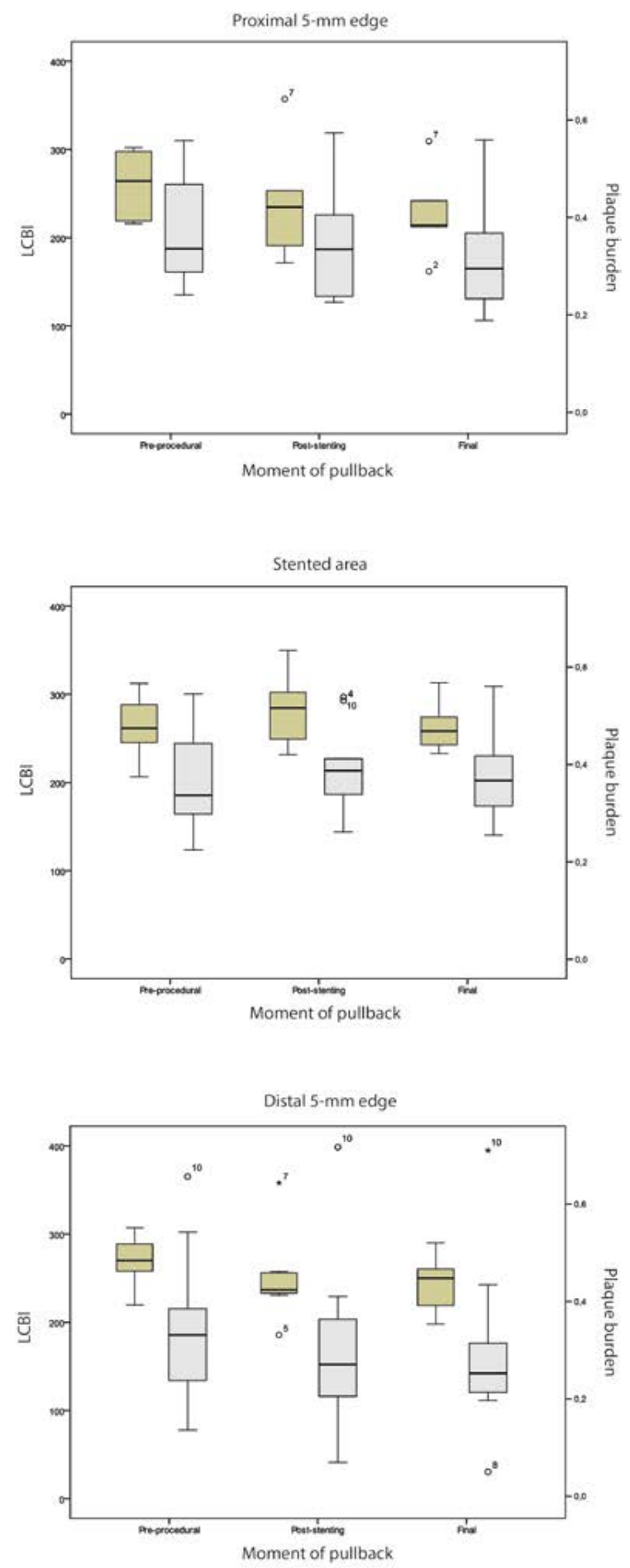

Figure 2: $\mathrm{LCBI}$ and plaque volume change during $\mathrm{PCI}$. Boxplot of $\mathrm{LCBI}$ and plaque burden changes from pre-procedural, post-stenting and final in the proximal stent edge (above); the stented area (middle) and the distal stent edge (below). In all three panels plaque burden (gray) remained constant while the LCBI (green) changes and decreases significantly in the stented area (middle). 


\begin{tabular}{|c|c|c|c|c|c|}
\hline & & Pre-stent & Post-stent & Final & P-value \\
\hline \multicolumn{6}{|l|}{ QCA outcomes } \\
\hline MLD, mm & & $0.9 \pm 0.4$ & $2.2 \pm 0.2$ & $3.0 \pm 0.4$ & $<0.001$ \\
\hline $\mathrm{RVD}, \mathrm{mm}$ & & $3.3 \pm 0.6$ & $3.3 \pm 0.4$ & $3.6 \pm 0.4$ & 0.007 \\
\hline DS, $\%$ & & $70.5 \pm 15.2$ & $33.3 \pm 8.4$ & $15.2 \pm 7.6$ & 0 \\
\hline $\mathrm{D} \max , \mathrm{mm}$ & & $3.9 \pm 0.9$ & & & \\
\hline \multicolumn{6}{|l|}{ IVUS-outcomes } \\
\hline \multirow[t]{3}{*}{$\mathrm{MLA}, \mathrm{mm}^{2}$} & Proximal $5 \mathrm{~mm}$ & $9.7 \pm 3.6$ & $9.9 \pm 4.6$ & $10.5 \pm 3.8$ & 0.59 \\
\hline & Stented area & $5.2 \pm 1.2$ & $4.7 \pm 1.2$ & $8.4 \pm 2.4$ & $<0.001$ \\
\hline & Distal $5 \mathrm{~mm}$ & $10.5 \pm 4.3$ & $9.9 \pm 4.6$ & $10.2 \pm 3.9$ & 0.9 \\
\hline \multirow[t]{3}{*}{ EEM CSA, $\mathrm{mm}^{2}$} & Proximal $5 \mathrm{~mm}$ & $22.7 \pm 5.8$ & $23.2 \pm 5.3$ & $22.0 \pm 4.7$ & 0.31 \\
\hline & Stented area & $20.6 \pm 4.6$ & $21.1 \pm 4.8$ & $21.6 \pm 4.5$ & 0.23 \\
\hline & Distal $5 \mathrm{~mm}$ & $20.4 \pm 5.4$ & $19.9 \pm 5.9$ & $19.4 \pm 5.7$ & 0.23 \\
\hline \multirow[t]{3}{*}{ Lumen CSA, mm² } & Proximal $5 \mathrm{~mm}$ & $12.3 \pm 3.6$ & $13.6 \pm 4.7$ & $13.4 \pm 3.6$ & 0.17 \\
\hline & Stented area & $10.8 \pm 2.8$ & $10.5 \pm 3.4$ & $11.5 \pm 3.0$ & 0.04 \\
\hline & Distal $5 \mathrm{~mm}$ & $11.3 \pm 3.4$ & $11.8 \pm 4.4$ & $11.6 \pm 3.7$ & 0.75 \\
\hline \multirow[t]{3}{*}{$\mathrm{P} \& \mathrm{M}$ CSA, $\mathrm{mm}^{2}$} & Proximal $5 \mathrm{~mm}$ & $10.6 \pm 3.2$ & $10.1 \pm 3.3$ & $9.5 \pm 3.1$ & 0.17 \\
\hline & Stented area & $10.2 \pm 2.9$ & $10.7 \pm 2.5$ & $10.5 \pm 2.5$ & 0.44 \\
\hline & Distal $5 \mathrm{~mm}$ & $9.9 \pm 3.3$ & $8.9 \pm 3.6$ & $8.7 \pm 3.6$ & 0.21 \\
\hline \multirow[t]{3}{*}{ Plaque burden, $\%$} & Proximal $5 \mathrm{~mm}$ & $0.46 \pm 0.1$ & $0.42 \pm 0.1$ & $0.40 \pm 0.1$ & 0.18 \\
\hline & Stented area & $0.48 \pm 0.1$ & $0.51 \pm 0.1$ & $0.48 \pm 0.0$ & 0.14 \\
\hline & Distal $5 \mathrm{~mm}$ & $0.45 \pm 0.0$ & $0.41 \pm 0.1$ & $0.40 \pm 0.0$ & 0.23 \\
\hline \multicolumn{6}{|l|}{ NIRS outcomes } \\
\hline \multirow[t]{3}{*}{ LCBI } & Proximal $5 \mathrm{~mm}$ & $92.8 \pm 131.9$ & $80.0 \pm 150.8$ & $65.3 \pm 119.5$ & 0.08 \\
\hline & Stented area & $160.0 \pm 95.6$ & $31.8 \pm 48.5$ & $20.7 \pm 34.9$ & 0.001 \\
\hline & Distal $5 \mathrm{~mm}$ & $48.0 \pm 53.7$ & $35.1 \pm 74.2$ & $20.4 \pm 40.2$ & 0.49 \\
\hline
\end{tabular}

the proximal $5 \mathrm{~mm}$ edge segment remained similar from pre-stent to post-stent $(93 \pm 132$ vs. $80 \pm 151)$, and decreased after final post-dilatation $(65 \pm 120)$.

The plaque and media CSA in the stented segment remained relatively constant in the stented area $\left(10.2 \pm 2.9 \mathrm{~mm}^{2}\right.$ pre-stenting vs. $10.7 \pm 2.5 \mathrm{~mm}^{2}$ poststenting vs. $10.5 \pm 2.5 \mathrm{~mm}^{2}$ after final post-dilatation $(\mathrm{p}=0.44)$ (Figure 2). The plaque burden in the stented segment remained relatively constant in the stented area $(0.48 \pm 0.1$ pre-stenting $v$ s. $0.51 \pm 0.1$ post-stenting vs. $0.48 \pm 0.0$ after final post-dilatation $(\mathrm{p}=0.14)$. In 1 patient we were unable to assess the proximal edge throughout the procedure due to the treatment of an ostial SVG lesion, in 1 patient the IVUS-NIRS of the proximal edge post-stenting failed due to technical error and in 1 patient the IVUS-NIRS of the distal edge pre-stenting failed due to an inability of the catheter to cross the lesion.

\section{Discussion}

With performing serial IVUS-NIRS we demonstrated a significant decrease of LCBI in the stented segment from pre-stenting to post-stenting and after post-dilatations while the plaque burden remained relatively unaltered throughout the procedure with the self-apposing stent.

The presence of large coronary LRPs in the target vessel segment could be a challenge during PCI, since it increases risk of peri-procedural complications. Modification of LRPs is stated to occur alongside coronary revascularization with the release of lipid contents and atherosclerotic debris. Plaque modification and shifting could lead to distal embolization of lipid rich particles potentially resulting in the obstruction of (small) side branches, microvascular obstruction and peri-procedural myocardial infarction $[12,13]$. A previous study using IVUS during PCI revealed that stent implantation not only causes plaque redistribution, plaque reduction or compression into the distal segments, but that the plaque shifts to the proximal reference segments as well [14]. Decrease of lipid content during PCI quantified by the LCBI is previously investigated in patients presenting with acute coronary syndrome as well in stable patients. Moreover, Maini et al. found a correlation of LCBI decrease and post-procedural troponin raise, and on occasion shifting of the LRP to the proximal and distal zones [15]. Our data demonstrates that, within the stented segment, the 
plaque burden remains relatively unchanged during the procedure (even after balloon post-dilatations) but that the LCBI decreases significantly. The LCBI decrease in the proximal $5 \mathrm{~mm}$ edge and in the stented area with the decrease in the distal $5 \mathrm{~mm}$ edge throughout the PCI without concomitant plaque burden decrease suggests that there is a shift of lipid alongside each step of coronary revascularization. Since the unaltered plaque burden suggests that the plaque content stays beyond the stent, which on its turn implies that the LCBI decrease during PCI might not necessarily be distal embolization of cholesterol or lipid particles. In one patient from our analyses, a distal protection device was used during PCI. Pathological specimen revealed only thrombus material, there was no lipid present in the distal protection device, supporting our hypothesis that the lipid content shifts. The discrepancy between plaque burden and lipid content changes can possibly be explained by significant decreased in NIRS signal (ability of NIRS to detect lipid) as a function of increasing distance to the vessel wall in larger vessels as the lipid content shifts outwards where as a function of distance the ability of NIRS to detect lipid simply decreases.

The patients in our analyses had a mean RVD of $3.3 \pm 0.6 \mathrm{~mm}$ which implies relatively large coronary arteries. The studied reference vessel diameter in the previous NIRS studies was not consequently reported. Maini et al. did not report the vessels RVDs. One of the autopsy validation studies of the NIRS technique reported a mean lumen diameter of $2.4 \mathrm{~mm}$ [16]. The in vivo validation study of the catheter based NIRS system in patients did not report any RVDs [17]. This can be explained by the fact that the IVUS NIRS technique has not yet been validated in larger RVDs and that our observations suggest a potential artefact, due to the relatively large distance from the catheter to the vessel wall and the size of the coronary artery. Larger NIRS studies with large reference vessel diameters, catheter eccentricity, and the whole vessel segment analyses are warranted to gain more insights in the lipid content modification, as well as the reliability of NIRS, during PCI of large coronary vessels.

\section{$\mathrm{PCl}$ using the self-apposing stent device}

High balloon pressures during PCI, which are often needed to ensure optimal stent expansion, may cause injuries to the arterial wall, lead to LRP shifts throughout the procedure or to lipid core penetration by stent struts. Iatrogenic injury of the internal elastic membrane can induce arterial inflammation which is associated with an increased neo-intimal growth and thus a larger risk of adverse events [18,19]. The use of the self-apposing stent could theoretically lead to reduced procedural pressures inserted to the coronary vessel wall, and therefore reduce the risk of potential injury. In the present study, all STENTYS stents were implanted with maximal balloon pressures of $11.4 \pm 3.8 \mathrm{~atm}$. The procedural LCBI shift we observed in the stented segments might be explained by the fact that procedural manoeuvre even with relatively low pressures could impact lipid content shift. It could also support the assumption of a possible miss-registration due to decreased signal and registration of the lipids by the NIRS catheter in relatively larger RVDs since the target vessel segments were relatively large in this study.

\section{Study Limitations}

This prospective observational study is limited by its small, single-center study design with no control group. The number of patients was too small to have power, thus the results should be interpreted with caution and should be hypothesis generating. However the serial INUS-NIRS assessment could give us specific insight in the plaque and lipid shift throughout PCI. Larger cohorts are warranted to prove our assumption. Furthermore, distal protection devices were not routinely used.

\section{Conclusion}

The current serial IVUS-NIRS findings demonstrated a significant decrease of LCBI in the stented segment from pre-stenting to post-stenting and after postdilatations while plaque burden remained unaltered throughout the procedure of lesions located in large RVDs. This might be explained by a possible artefactual registration of NIRS in vessels with large RVDs. Additional research with larger numbers of IVUSNIRS analyses is warranted to confirm this concept. 


\section{Executive summary}

Background: Lipid rich plaque (LRP) content has been shown to decrease after high-pressure balloon-expandable stent placement. LRPs are quantified by the lipid core burden index (LCBI) on near-infrared spectroscopy (NIRS). We aim to evaluate the changes in LRP content during sequential steps of non-urgent percutaneous coronary intervention $(\mathrm{PCI})$ using low-pressures.

Methods: A total of 10 patients with a LCBI $>200$ on pre-stenting NIRS and successful PCI with the self-apposing stent were included. Quantitative coronary angiography was used to assess luminal diameters. IVUS-NIRS was performed pre-stenting after initial predilatation, post-stenting and after post-dilatation to assess the LCBI and the plaque burden to evaluate plaque modification. IVUSNIRS is analyzed for all stented segments including proximal and distal stent edges using dedicated software.

Results: Mean balloon pressure was $11.4 \pm 3.8 \mathrm{~atm}$. Pre-stent reference vessel diameter (RVD) was $3.3 \pm 0.6 \mathrm{~mm}$, Dmax was $3.9 \pm 0.9$ $\mathrm{mm}$. Mean LCBI of the stented segment was $160 \pm 96$ pre-stenting, $32 \pm 49$ post-stenting and $21 \pm 35$ after post-dilatation $(p=0.001)$. The plaque burden remained constant in the stented segment; $0.48 \pm 0.1 \mathrm{~mm}^{2}$ pre-stenting vs. $0.51 \pm 0.1 \mathrm{~mm}^{2}$ post-stenting vs. $0.48 \pm 0 \mathrm{~mm}^{2}$ after final post-dilatation $(\mathrm{p}=0.14)$.

Conclusions: Serial IVUS-NIRS demonstrated a significant decrease of LCBI in the stented segment from pre-stenting to poststenting and after post-dilatations, while plaque burden remained unaltered throughout PCI in relatively large RVDs. This discrepancy might possible explained by the fact that NIRS might be not detecting the lipid in larger RVDs since it is only validated in small RVDs. Research including larger numbers of patients are warranted to confirm this concept.

\section{References}

1. Farb A, Tang AL, Burke AP, et al. Sudden Coronary DeathFrequency of Active Coronary Lesions, Inactive Coronary Lesions, and Myocardial-Infarction. Circulation. 92(7): 1701-1709 (1995).

2. Kolodgie FD, Burke AP, Farb A, et al. The thin-cap fibroatheroma: a type of vulnerable plaque - The major precursor lesion to acute coronary syndromes. Cur. Opin. Cardiol. 16(5): 285-292 (2001).

3. Silvestre-Roig C, de Winther MP, Weber C, et al. Atherosclerotic Plaque Destabilization Mechanisms, Models, and Therapeutic Strategies. Circulation. Res. 114(1): 214-226 (2014).

4. Virmani R, Kolodgie FD, Burke AP, et al. Lessons from sudden coronary death-A comprehensive morphological classification scheme for atherosclerotic lesions. Arterioscler. Thrombo. Vascul. Biol. 20(5): 1262-1275 (2000).

5. Weber C, Noels H. Atherosclerosis: current pathogenesis and therapeutic options. Nature. Med. 17(11): 1410-1422 (2011).

6. Uetani T, Amano T, Ando H, et al. The correlation between lipid volumes in the target lesion, measured by integrated backscatter intravascular ultrasound, and post-procedural myocardial infarction in patients with elective stent implantation. Eur. Heart. J. 29(14): 1714-1720 (2008).

7. Kawaguchi R, Oshima S, Jingu M, et al. Usefulness of virtual histology intravascular ultrasound to predict distal embolization for ST-segment elevation myocardial infarction. J. Am. Coll. Cardiol. 50(17): 1641-1646 (2007).

8. Kawamoto T, Okura H, Koyama Y, et al. The relationship between coronary plaque characteristics and small embolic particles during coronary Stent implantation. J. Am. Coll. Cardiol. 50(17): 1635-1640 (2007).

9. Pervaiz MH, Sood P, Sudhir K, et al. Periprocedural Myocardial Infarction in a Randomized Trial of Everolimus-Eluting and Paclitaxel-Eluting Coronary Stents Frequency and Impact on Mortality According to Historic Versus Universal Definitions. Circulation. Cardiovasc. Interven. 5(2): 150-156 (2012).

10. Ricciardi MJ, Wu E, Davidson CJ, et al. Visualization of discrete microinfarction after percutaneous coronary intervention associated with mild creatine kinase-MB elevation. Circulation. 103(23): 2780-2783 (2001).
11. Puri R, Madder RD, Madden SP, et al. Near-Infrared Spectroscopy Enhances Intravascular Ultrasound Assessment of Vulnerable Coronary Plaque: A Combined Pathological and In Vivo Study. Arterioscler. Thromb. Vasc. Biol. 35(11): 2423-2431 (2015).

12. Goldstein JA, Maini B, Dixon SR, et al. Detection of lipidcore plaques by intracoronary near-infrared spectroscopy identifies high risk of periprocedural myocardial infarction. Circ. Cardiovasc. Interv. 4(5): 429-437 (2011).

13. Matsuo K, Ueda Y, Tsujimoto M, et al. Ruptured plaque and large plaque burden are risks of distal embolisation during percutaneous coronary intervention: evaluation by angioscopy and virtual histology intravascular ultrasound imaging. EuroIntervention. 9(2): 235-242 (2013).

14. Ahmed JM, Mintz GS, Weissman NJ, et al. Mechanism of lumen enlargement during intracoronary stent implantation: an intravascular ultrasound study. Circulation. 102(1): 7-10 (2000).

15. Maini A, Buyantseva L, Maini B. In vivo lipid core plaque modification with percutaneous coronary revascularization: a near-infrared spectroscopy study. J. Invasive. Cardiol. 25(6): 293-295 (2013).

16. Gardner CM, Tan HW, Hull EL, et al. Detection of Lipid Core Coronary Plaques in Autopsy Specimens with a Novel CatheterBased Near-Infrared Spectroscopy System. JACC. Cardiovasc. Imag. 1(5): 638-48 (2008).

17. Waxman S, Dixon SR, L'Allier P, et al. In vivo validation of a catheter-based near-infrared spectroscopy system for detection of lipid core coronary plaques: initial results of the SPECTACL study. JACC. Cardiovasc. Imag. 2(7): 858-868 (2009).

18. Frobert O, Sarno G, James SK, et al. Effect of stent inflation pressure and post-dilatation on the outcome of coronary artery intervention. A report of more than 90,000 stent implantations. PLoS. One. 8(2): e56348 (2013).

19. Hoffmann R, Guagliumi G, Musumeci G, et al. Vascular response to sirolimus-eluting stents delivered with a nonaggressive implantation technique: comparison of intravascular ultrasound results from the multicenter, randomized E-SIRIUS, and SIRIUS trials. Catheter. Cardiovasc. Interv. 66(4): 499-506 (2005). 